\section{Nervenschmerzen: oft unerkannt und schlecht therapiert}

Die neue Leitlinie „Neuropathischer Schmerz" gibt einen Überblick über die aktuell eingesetzten Diagnosemethoden und darüber, welche therapeutischen Möglichkeiten es gibt und wie effektiv sie sind (Schlereth T: Diagnose und nichtinterventionelle Therapie neuropathischer Schmerzen-Leitlinien für Diagnostik und Therapie in der Neurologie. Herausgegeben von der Kommission Leitlinien der Deutschen Gesellschaft für Neurologie. 4. September 2019).

Etwa 3 bis 5 Prozent der Bevölkerung in Deutschland leiden an Nervenschmerzen. Die wohl bekanntesten Ursachen für neuropathische Schmerzen sind die Gürtelrose (Herpes zoster), die als Spätfolge einer Windpockeninfektion auftreten kann, sowie der Diabetes mellitus, bei dem die Nerven durch den erhöhten Blutzuckerspiegel geschädigt werden. „Neuropathische Schmerzen können aber beispielsweise auch nach einem Schlaganfall oder infolge einer Multiplen Sklerose auftreten, oder auch genetisch bedingt sein“, erklärt Professor Dr. med. Christian Maihöfner, Kongresspräsident des Deutschen Schmerzkongresses 2019 und Chefarzt der Neurologischen Klinik am Klinikum Fürth.

Die Therapie neuropathischer Schmerzen unterscheidet sich von der Therapie anderer chronischer Schmerzen, bei denen das Nervensystem nicht geschädigt ist. Wie nun die verschiedenen Schmerzarten diagnostisch klar voneinander abgegrenzt werden und welche Behandlungsmöglichkeiten nach aktuellem wissenschaftlichem Stand geeignet sind, zeigt die neue S2k-Leitlinie, an deren $\mathrm{Er}$ stellung Maihöfner beteiligt war. Für die Diagnose ist eine ausführliche Befragung des Patienten ebenso essentiell wie die klinische Untersuchung. Um Art und Ausmaß der Schädigung zu identifizieren, werden neben neurophysiologischen Testmethoden zunehmend auch bildgebende Verfahren wie Kernspinto- mographie und Ultraschall eingesetzt. „Als Goldstandard für den Nachweis einer Schädigung der kleinen, schmerzleitenden Fasern kann aber nach wie vor die Hautbiopsie gelten“, sagt Maihöfner.

Eine ursächliche Therapie der neuropathischen Schmerzen ist oft nicht möglich. Aber die Experten der Leitlinie betonen, dass die Möglichkeiten einer heilenden oder kausalen Therapie ausgeschöpft werden sollten. „Bei Nervenschmerzen, die infolge einer diabetischen Neuropathie auftreten, muss versucht werden, den Diabetes optimal einzustellen“, erläutert Maihöfner.

Die Behandlung mit Schmerzmedikamenten sei nach wie vor eine Herausforderung, denn man kann selten vorhersagen, welches Medikament bei welchem Patienten wirkt. Auch können Nebenwirkungen auftreten, und bei manchen Patienten kann keine ausreichende Wirkung erreicht werden, so der Experte. Die Leitlinie macht klare, wissenschaftlich begründete Aussagen zur Wirksamkeit der jeweiligen Medikamente und gibt Empfehlungen zur medikamentösen Therapie, betont aber auch, dass neben der medikamentösen Behandlung die psychosozialen Umstände der Patienten berücksichtigt werden müssen.

Als wichtige Therapieoption gilt nach wie vor die sog. multimodale Schmerztherapie, bei der die Patienten neben Ergotherapie und Physiotherapie auch eine Schmerzpsychotherapie erhalten sollten. „Im Rahmen dieser interdisziplinären Behandlung lernen die Patienten, mit dem Schmerz besser umzugehen und sogenannte Coping-Strategien zu entwickeln“, sagt Professor Dr. med. Claudia Sommer, Präsidentin der Deutschen Schmerzgesellschaft e.V. und Leitende Oberärztin und Schmerzforscherin an der Neurologischen Klinik und Poliklinik des Uniklinikums Würzburg. Schmerzbewältigungstechniken und das Überwinden eines Vermeidungsverhaltens können erlernt werden. Denn entscheidend für den Therapieerfolg sind das individuelle Schmerzempfinden und das Lebensgefühl des Patienten, betont Sommer. „Ziel muss es sein, die Lebensqualität wieder zu verbessern, soziale Aktivitäten zu ermöglichen und das soziale Beziehungsgefüge sowie die Arbeitsfähigkeit zu erhalten.“

- https://www.dgn.org/images/

red_leitlinien/LL_2019/

PDFs_Download/030114_LL_Neuro-

pathische_Schmerzen_2019.pdf.

Nach einer Mitteilung der Kongress-Pressestelle des Deutschen Schmerzkongress 2019 